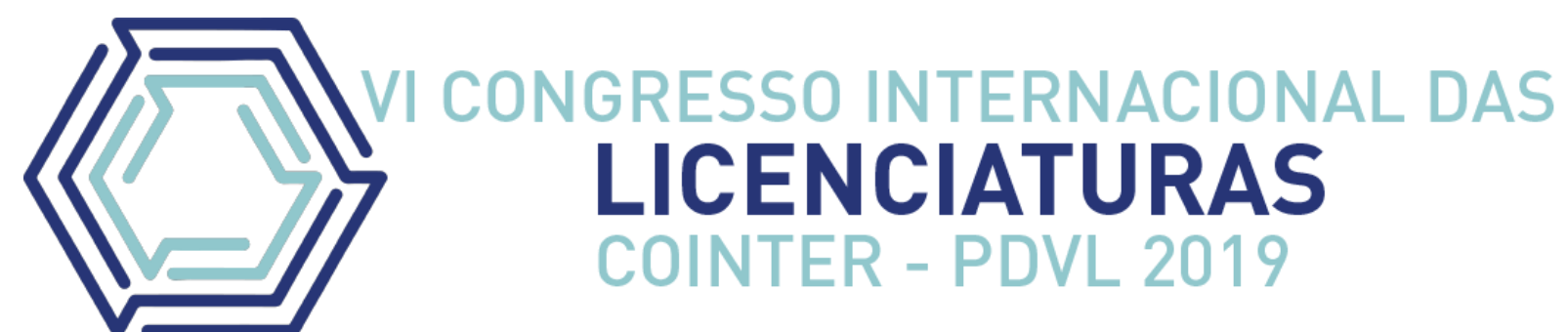

\title{
ETNOBOTÂNICA: O ESTUDO DAS PLANTAS MEDICINAIS DA CAATINGA NO ENSINO MÉDIO
}

\author{
ETNOBOTÁNICA: EL ESTUDIO DE LAS PLANTAS MEDICINALES DE \\ CAATINGA EN LA ESCUELA SECUNDARIA
}

\section{ETHNOBOTANY: THE STUDY OF CAATINGA'S MEDICAL PLANTS IN HIGH SCHOOL}

\author{
Apresentação: Comunicação Oral \\ Geovana Maria da Conceição Neto ${ }^{1}$; Aretuza Bezerra Brito Ramos²; Geraldo Martins de \\ Oliveira Júnior ${ }^{3}$
}

DOI: $\underline{\text { https://doi.org/10.31692/2358-9728.VICOINTERPDVL.2019.0131 }}$

\begin{abstract}
Resumo
A Etnobotânica apresenta-se como uma ciência que tem por finalidade o estudo e análise da relação entre o ser humano e as plantas, destacando-se como uma importante ferramenta cultural. Destarte, este trabalho objetivou analisar a percepção dos estudantes do ensino médio acerca da utilização de plantas medicinais da Caatinga no tratamento de patologias. O estudo foi realizado em Serrita-PE, tendo como público alvo o corpo discente do $2^{\circ}$ ano A do ensino médio da Escola EREM Desembargador João Paes. Foram realizadas entrevistas como préteste e pós-teste a partir da aplicação de questionários semiestruturados, além do desenvolvimento de uma aula expositiva e dialogada sobre plantas medicinais. A partir da análise dos dados, pode-se constatar que $69,7 \%$ dos entrevistados residem na zona urbana. No que se refere ao conhecimento das plantas medicinais, todos afirmaram conhecer essas plantas e que fazem e/ou algum familiar faz uso. Após a realização da intervenção foi possível observar que as espécies medicinais mais citadas são nativas da Caatinga, demonstrando um número relativamente alto quando comparado ao obtido no pré-teste. Os estudantes destacaram que existe uma ligação de uso e compartilhamento de conhecimentos sobre essas plantas, por serem parte integrante e cultural da comunidade. Além disso, os entrevistados afirmaram que os estudos etnobotânicos são importantes para a obtenção de conhecimentos acerca do uso correto dessas plantas, tais como o preparo, a quantidade e a dosagem. Desta maneira, pode-se concluir que a utilização de aulas expositivas e dialogadas se estabelecem como um excelente instrumento de ensino-aprendizagem, principalmente no que se refere à temáticas que fazem parte do seu cotidiano, destacando que com o estudo realizado foi possível observar uma sensível incorporação desses conhecimentos.
\end{abstract}

\footnotetext{
${ }^{1}$ Ciências Biológicas, FACHUSC, geoneto1998@gmail.com.

${ }^{2}$ Mestre em Gestão e Políticas Ambientais, UFPE, aretuzab@yahoo.com.br.

${ }^{3}$ Mestrando em Ensino de Biologia, UFPE, geraldomarthins@hotmail.com.
} 
Palavras-Chave: Estudos Etnobotânicos, Aula Expositiva e Dialogada, Ensino-aprendizagem. Resumen

La etnobotánica se presenta como una ciencia cuyo propósito es el estudio y análisis de la relación entre los seres humanos y las plantas, destacándose como una importante herramienta cultural. Por lo tanto, este estudio tuvo como objetivo analizar la percepción de los estudiantes de secundaria sobre el uso de plantas medicinales Caatinga en el tratamiento de patologías. El estudio se realizó en Serrita-PE, teniendo como público objetivo a los alumnos del segundo año de secundaria de la escuela EREM Desembargador João Paes. Las entrevistas se realizaron como pretest y posttest a partir de la aplicación de cuestionarios semiestructurados, así como el desarrollo de una conferencia y un diálogo sobre plantas medicinales. Del análisis de datos, se puede ver que el $69.7 \%$ de los encuestados viven en el área urbana. Con respecto al conocimiento de las plantas medicinales, todos dijeron que saben sobre estas plantas y lo que hacen y / o algún miembro de la familia utiliza. Después de realizar la intervención, fue posible observar que las especies medicinales más citadas son nativas de Caatinga, mostrando un número relativamente alto en comparación con el obtenido en la prueba preliminar. Los estudiantes señalaron que existe un vínculo entre el uso y el intercambio de conocimientos sobre estas plantas, ya que son una parte integral y cultural de la comunidad. Además, los encuestados declararon que los estudios etnobotánicos son importantes para obtener conocimiento sobre el uso correcto de estas plantas, como la preparación, la cantidad y la dosis. Por lo tanto, se puede concluir que el uso de conferencias y diálogos se establece como un excelente instrumento de enseñanza-aprendizaje, especialmente con respecto a los temas que forman parte de su vida cotidiana, destacando que con el estudio fue posible observar un incorporación sensible de este conocimiento.

Palabras Clave:Estudios etnobotánicos, conferencias y diálogos, enseñanza-aprendizaje.

\begin{abstract}
Ethnobotany presents itself as a science whose purpose is the study and analysis of the relationship between human beings and plants, standing out as an important cultural tool. Thus, this study aimed to analyze the perception of high school students about the use of Caatinga medicinal plants in the treatment of pathologies. The study was conducted in SerritaPE, having as target audience the students from the 2nd year of high school of the EREM Desembargador João Paes School. Interviews were conducted as pretest and posttest from the application of semi-structured questionnaires, as well as the development of a lecture and dialogued about medicinal plants. From the data analysis, it can be seen that $69.7 \%$ of respondents live in the urban area. Regarding the knowledge of medicinal plants, all said they know about these plants and what they do and / or some family member makes use of. After performing the intervention it was possible to observe that the most cited medicinal species are native to Caatinga, showing a relatively high number when compared to the one obtained in the pretest. Students pointed out that there is a link of use and sharing of knowledge about these plants, as they are an integral and cultural part of the community. In addition, respondents stated that ethnobotanical studies are important for gaining knowledge about the correct use of these plants, such as preparation, quantity and dosage. Thus, it can be concluded that the use of lectures and dialogues are established as an excellent teachinglearning tool, especially regarding the themes that are part of their daily lives, highlighting that with the study it was possible to observe a sensitive incorporation of this knowledge.
\end{abstract}

Keywords: Ethnobotanical Studies, Lecture and Dialogue, Teaching-learning. 


\section{Introdução}

A Enobotânica é a ciência que tem por finalidade, estudar e analisar a relação entre pessoas e plantas (MARTINS et al., 2005). Esta área do conhecimento constitui-se em uma ferramenta de pesquisa que auxilia a interação do homem com a diversidade vegetal e contribui de modo significativo nas enfermidades humanas, nos rituais religiosos e até mesmo em padrões de alimentação. Como consequência da grande conexão que se estabelece entre o saber popular e a ciência propriamente dita, a Etnobotânica tem ganhado destaque no âmbito científico (FRANCO et al., 2011).

Ademais, a vegetação da Caatinga é caracterizada como um ecossistema que apresenta florestas secas, sendo composta por árvores e arbustos baixos com características xerofíticas, sendo alvo da exploração humana, devido à atividade agrícola desenvolvida, pelo uso da pecuária extensiva e pelo extrativismo na extração de lenha e madeira. Outrossim, a Caatinga é o bioma exclusivo do Nordeste Brasileiro, apresentando um elevado índice de diversidade e endemismo, tornando-se necessário um melhor conhecimento de sua flora para possíveis medidas de conservação de suas áreas (PRADO, 2003).

Essa diversidade de plantas vem sendo utilizada pelo homem na produção de óleos, resina, borrachas, tanino, fármacos e cosméticos. Porém, há muito tempo o homem vem se adaptando aos períodos de seca, graças a sua convivência e integração com esse bioma. É mister frisar que a ligação entre o ser humano e as plantas é antiga, há muito tempo as populações humanas vêm aprendendo a usá-las, acumulando assim, conhecimentos sobre as utilizações dos vegetais para o seu proveito e transformando o ambiente em que vivem (RODRIGUES, 2007).

O uso de plantas medicinais para o tratamento, a cura e prevenção de doenças é uma das mais antigas formas de prática medicinal da humanidade (VEIGA; PINTO, 2005). Segundo Pereira (2005), essa prática está relacionada a uma quantidade significativa de espécies vegetais da Caatinga usadas para fins terapêuticos: aroeira, joazeiro, mororó, catingueira, angico, faveleira, jurema-preta, são alguns exemplos de plantas utilizadas nos processos inflamatórios, doenças intestinais e cicatrização de ferimentos.

A Educação Ambiental consiste em proporcionar teoricamente uma compreensão crítica, com a finalidade de desenvolver atitudes conscientes a respeito da conservação dos recursos naturais como forma de ressaltar uma melhor qualidade de vida para todos. Assim, deve ser contextualizada em diferentes espaços e perspectivas, promovendo a percepção do discente 
como ser integrante e participante do meio (BRANCALIONE, 2016).

Assim, destaca-se que conforme a relação do homem com a terra passa por uma constante transformação e o contato com centros urbanos aumenta, a rede de transmissão das informações sobre as plantas medicinais pode sofrer alterações, tornando-se necessário o resgate deste conhecimento e dos métodos de tratamento, como um meio de deixar registrado esse modo de aprendizagem informal (PILLA et al., 2006).

Desta forma, é notória a importância da abordagem sobre essas plantas no contexto de estudos, por ser um tema amplo e que pode ser trabalhado de diferentes maneiras, possibilitando conhecer melhor a variedade de plantas nativas e seus potenciais. Destarte, esse trabalho objetivou analisar a percepção dos estudantes do ensino médio acerca da utilização de plantas medicinais da Caatinga no tratamento de patologias.

\section{Fundamentação Teórica}

Os conhecimentos etnobotânicos já são há tempos utilizados em várias práticas e por diversas culturas (PAIVA, 2013). Os seres humanos tem se relacionado profundamente com o cultivo de plantas desde as suas primeiras associações, quando os quintais passaram a ser uma estratégia de cultivo dos seus próprios alimentos e remédios (NASCIMENTO, 2008).

Segundo Guarim-Neto, Santana e Silva (2000), é por meio da Etnobotânica que se busca o conhecimento e o resgate do saber botânico tradicional, principalmente relacionado ao uso dos recursos da flora. Os trabalhos desenvolvidos nessa área vêm aumentando, particularmente em países como o Brasil. O foco varia conforme a região, mas a temática acerca do uso das plantas medicinais está presente em todos eles (OLIVEIRA et al., 2009).

Não obstante, percebe-se que não existe tanto interesse das novas gerações por este conhecimento, o que contribui para o consequente desaparecimento de algumas espécies ou enorme dificuldade em encontrá-las. Diante disso, torna-se necessário uma maior valorização dessa cultura e reintegração do conhecimento que a população possui sobre o uso dos recursos naturais (MARTINS, 2012).

A flora brasileira dispõe de uma grande quantidade de ervas medicinais, e por ser tão rica algumas plantas e suas propriedades ainda não foram estudadas profundamente (MEDEIROS; CRISTOSTINO, 2013). Mas, devido a expansão das fronteiras agrícolas e urbanas, aos poucos, essa flora tem sido modificada por serem espécies nativas de alto potencial madeireiro e medicinal (QUEIROZ, 2011). 
Entre os biomas brasileiros, a Caatinga destaca-se por apresentar uma variedade de plantas medicinais já consagradas pela farmacopéia. $\mathrm{O}$ uso destes vegetais entre as populações nordestinas aponta um grande conhecimento acerca desses recursos ambientais, destacando um sistema próprio de suas utilizações. Estas comunidades apresentam uma vasta farmacopéia natural, onde boa parte dos recursos vegetais são encontrados nos ambientes naturais em que vivem, ou mantidos em ambientes de cultivo antrópico (MARTINS, 2012).

De acordo com Mera et al. (2018), as plantas medicinais são vegetais que possuem ações farmacêuticas com o efeito de curar ou amenizar algumas enfermidades. Além disso, realizam um papel muito importante na questão socioeconômico tanto para as populações que vivem no meio rural quanto no meio urbano, pois com a utilização dessas espécies para fins medicinais, na maioria das vezes nativas da sua região, ou cultivadas em quintais, é possível reduzir ou eliminar os gastos com medicamentos sintéticos.

O conhecimento acerca das plantas medicinais representa muitas vezes o único meio terapêutico de várias comunidades e grupos étnicos (MACIEL; PINTO; VEIGA, 2002). Porém, há uma preocupação quanto ao uso indevido que muitas pessoas fazem das plantas medicinais, pois muitas apresentam toxicidade elevada e devem ser utilizadas de forma correta, de preferência com acompanhamento médico (KOVALSKI; OBARA, 2013).

Em virtude de seus princípios ativos e sua eficácia, as instituições de ensino e pesquisa vêm aumentando os estudos sobre esses saberes (MERA et al., 2018), os quais são capazes de proporcionar caminhos férteis para a prática de Educação Ambiental, tendo as plantas medicinais como eficientes instrumentos pedagógicos, enquanto elementos que podem auxiliar a relação educativo-ambiental, oferecendo oportunidades de inserção de diferentes aspectos (GUARIM-NETO, 2006).

Para Silva (2012), é por meio da Educação Ambiental que se obtêm o conhecimento sobre a diversidade, mediante a um processo pedagógico interativo e permanente, que busca sensibilizar o educando e a comunidade, proporcionando o desenvolvimento de ações necessárias para a preservação, prevenção e melhoria de vida.

Ademais, destaca-se que a utilização da Etnobotânica como instrumento para o fomento ao diálogo entre os diferentes saberes de ensino/aprendizagem de Biologia possibilita a interação do homem com o meio natural, destacando que além dos conhecimentos científicos, os saberes populares também apresentam-se como importante ferramenta de desenvolvimento social (BAPTISTA, 2007). 


\section{Metodologia}

A presente pesquisa foi realizada em Serrita, (Figura 1), município do Sertão Pernambucano, pertencente a microrregião de Salgueiro apresentando uma população de aproximadamente 18.331 habitantes, distribuídos em uma área de $1.538,497 \mathrm{Km}^{2}$. Sua densidade demográfica é de 11,92 hab/km² (IBGE, 2010), com clima semiárido.

Figura 1 - Mapa de localização do Município de Serrita Pernabuco. Fonte: Modificado de Mapas para Colorir, 2019.

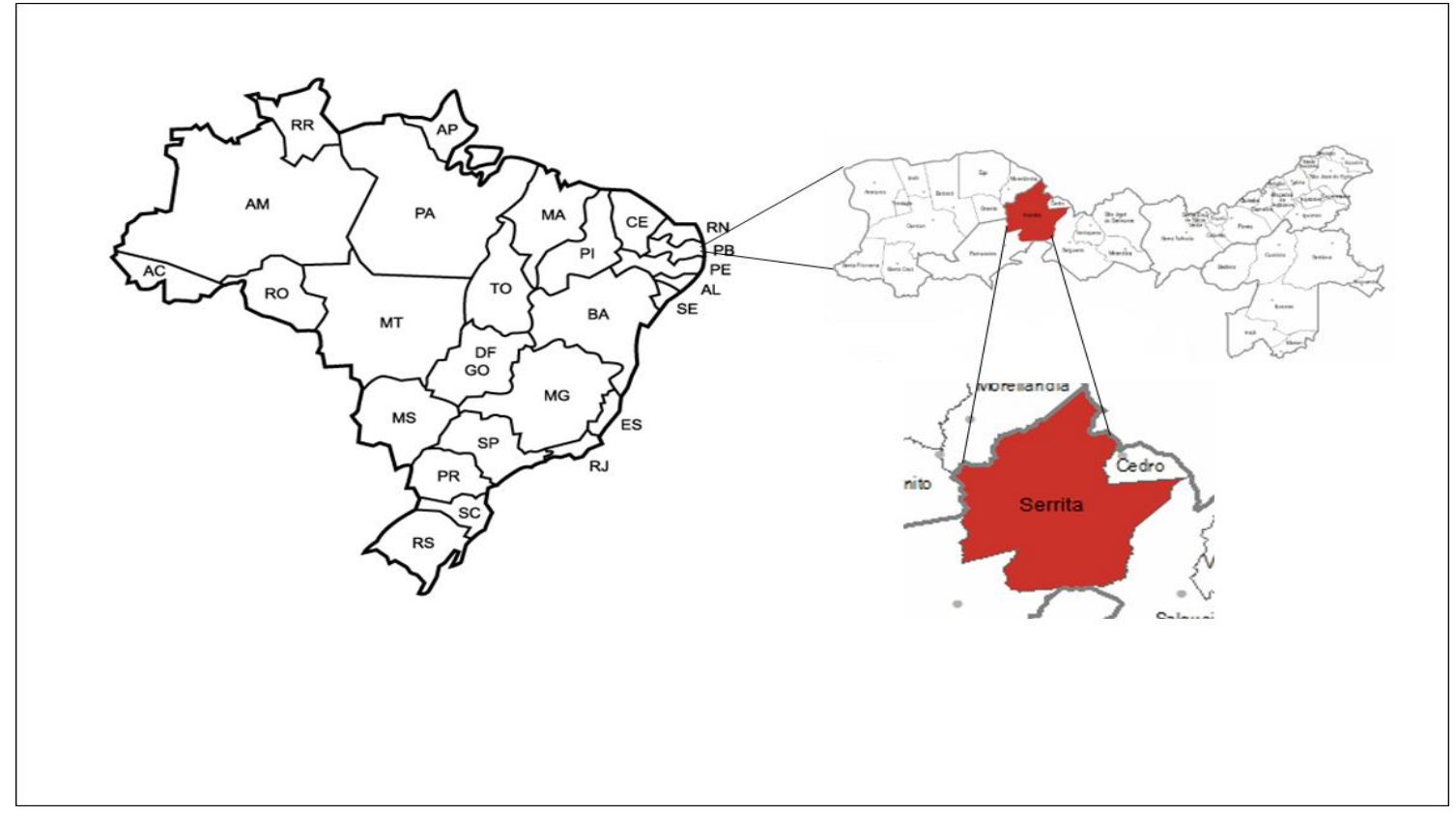

A área foco deste estudo foi a Escola EREM Desembargador João Paes, instituição de ensino médio semi-integral, a qual atende a maioria dos discentes do município. Ademais, o público alvo foi o corpo discente do $2^{\circ}$ ano A (33 participantes) do ensino médio, cujo critério usado para escolha da turma se deu pela disposição das aulas, sendo a única com aulas geminadas. Para o desenvolvimento desta pesquisa sucedeu-se ações metodológicas, em que foram realizadas entrevistas semiestruturadas como pré-teste e pós-teste, e também o desenvolvimento de uma atividade a partir do embasamento teórico sobre o tema proposto, com utilização de recursos para a condução da mesma em sala de aula.

Como forma de compreender, enfatizar e mensurar o objetivo proposto pela pesquisa optou-se pela abordagem quali-quantitativa e do tipo estudo de caso exploratório que teve como intuito a realização de descobertas. A pesquisa foi desenvolvida a partir de três etapas, em que a primeira consistiu na realização de uma entrevista como pré-teste contendo 11 
perguntas, sendo seis objetivas e cinco subjetivas, e que teve como propósito averiguar os conhecimentos prévios dos estudantes acerca das plantas medicinais. A primeira pergunta indagava sobre o local onde residem os entrevistados, a segunda tratava do conhecimento sobre as plantas medicinais, a terceira interrogava a respeito do uso dessas plantas e a quarta sobre o motivo pela as quais são usadas. Na quinta pergunta questionou-se sobre as plantas que os participantes mais utilizavam como medicinal, na sexta a intenção foi saber sobre quais entre as plantas citadas eram nativas da Caatinga e na sétima interrogou-se a frequência de uso das mesmas. A oitava pergunta indagava sobre o efeito do uso das plantas medicinais, a nona tratava das formas de uso dessas plantas, a penúltima questionou sobre a importância dessas plantas e a décima - primeira e última pergunta a respeito da transmissão de informações sobre as plantas com fim medicinal, (Apêndice A).

$\mathrm{Na}$ segunda etapa, ocorreu o embasamento teórico sobre as características e particularidades das plantas medicinais em uma aula expositiva e dialogada, em que foram ocasionados momentos de discussões que sucedeu-se através de recursos tecnológicos e amostras de plantas. Os educandos tiveram o primeiro contato com os conceitos acerca da temática, por meio da exibição de imagens, vídeos e textos que teve como propósito ocasionar análise, reflexão e despertar nos mesmos o interesse pelo conhecimento científico do tema abordado. Em sequência foram distribuídas amostras de plantas medicinais nativas, com o intuito de evidenciar e garantir maior segurança e eficácia no manuseio e uso das mesmas.

$\mathrm{Na}$ terceira e última etapa, realizou-se uma entrevista como pós-teste contendo cinco perguntas, sendo todas subjetivas, bem como uma sondagem para analisar a eficácia do procedimento metodológico por meio do grau de conhecimentos e conscientização adquiridos pelos educandos sobre a temática trabalhada. A primeira pergunta tratava sobre as plantas mais utilizadas pelos entrevistados como medicinais, a segunda questionava sobre quais das plantas citadas eram nativas da Caatinga, a terceira indagava a respeito da relação dessas plantas com a comunidade a qual os entrevistados estão inseridos, a penúltima questionava sobre a importância de buscar informações através dos conhecimentos dos mais velhos e a quinta e última pergunta buscou saber como essas informações ajudam no processo de aprendizagem sobre as plantas medicinais, (Apêndice B).

A avaliação do desenvolvimento das ações metodológicas ocorreu de forma contínua, durante todas as etapas e em todos os momentos, e teve como intuito verificar a participação e principalmente o interesse dos estudantes. 
As espécies botânicas citadas pelos entrevistados foram listadas e após o levantamento, identificadas conforme o sistema de classificação Angiosperm Phylogny Group - APG II e a sua nomenclatura seguiu o apresentado pelo Centro Nordestino de Informações sobre Plantas - CNIP, e pelo guia ilustrado para identificação das famílias de fanerógamas nativas e exóticas no Brasil, baseado no APG II (SOUZA; LORENZI, 2008).

\section{Resultados e Discussão}

A partir da análise dos dados, pode-se constatar que dentre os 33 estudantes entrevistados a maioria $(69,7 \%)$ residem na zona urbana. No que se refere ao conhecimento das plantas medicinais, todos afirmaram conhecer essas plantas e que fazem e/ou algum familiar faz uso. Tais resultados corroboram com a observação feita por Kovaski e Obara (2013), quando ressaltam a importância do conhecimento sobre as plantas medicinais que os envolvidos ou a comunidade já detêm, sendo que esses fazem uso em seu cotidiano.

Quanto ao motivo pela as quais são usadas, os entrevistados relataram ser para cura ou tratamento de alguma doença, corroborando assim com Veiga e Pinto (2005), onde afirmam que o uso de plantas medicinais para o tratamento, cura ou prevenção de doenças são algumas das mais antigas formas de prática medicinal da humanidade.

Com relação as plantas mais utilizadas pelos entrevistados com fins medicinais, foram mencionadas 25 espécies distribuídas em 17 famílias botânicas, em que Fabaceae apresentou um maior número, (Tabela 1). Dentre essas plantas, destacaram-se Capim-santo (Cymbopogon citratus Stapf.) (14 citações), Erva doce (Pimpinella anisum L.) (13), Alecrim (Rosmarinus officialis L.) e Hortelã (Mentha spp.) ambas citadas 11 vezes. Percebe-se que resultados semelhantes foram observados em um trabalho realizado por Rezende e Cocco (2002), e que segundo Caravaca (2000), cada povo tem sua própria lista das plantas medicinais mais comuns da região em que habitam. A família Fabaceae também se destacou no trabalho de Ribeiro et al. (2014).

Tabela 1. Espécies indicadas como medicinais pelos participantes do ensino médio da Escola EREM Desembargador João Paes, Serrita/PE.

\begin{tabular}{|l|c|}
\hline \multicolumn{1}{|c|}{ Família/ Espécie } & Nome Vernáculo \\
\hline AMARANTHACEAE & Mentruz \\
\hline Chenopodium ambosioides & \\
\hline ANACARDIACEAE & \\
\hline
\end{tabular}




\begin{tabular}{|c|c|}
\hline Myracrodruon urundeuva (Alemão) Engl. & Aroeira \\
\hline \multicolumn{2}{|l|}{ APIACEAE } \\
\hline Anethum graveolens $\mathrm{L}$. & Endro \\
\hline Coriandrum sativum $\mathrm{L}$. & Coentro \\
\hline Pimpinella anisum $\mathrm{L}$. & Erva-doce \\
\hline \multicolumn{2}{|l|}{ ASTERACEAE } \\
\hline Achyrocline satureioides (Lam) DC & Marcela \\
\hline Matricaria recutita $\mathrm{L}$. & Camomila \\
\hline \multicolumn{2}{|l|}{ BURSERACEA } \\
\hline Commiphora leptophloeos (Mart.) J. B. gillett & Imburana-de-cambão \\
\hline \multicolumn{2}{|l|}{ BORAGINACEAE } \\
\hline Heliotropium indicum $\mathrm{L}$. & Crista-galo \\
\hline \multicolumn{2}{|l|}{ CACTACEAE } \\
\hline Cereus jamacuru DC. & Mandacaru \\
\hline \multicolumn{2}{|l|}{ CANNABACEAE } \\
\hline Cannabis sativa $\mathrm{L}$. & Maconha \\
\hline \multicolumn{2}{|l|}{ FABACEAE } \\
\hline Prosopis juliflora (Sw.) DC. & Algaroba \\
\hline \multicolumn{2}{|l|}{ LAMIACEAE } \\
\hline Melissa officinalis $\mathrm{L}$. & Erva-cidreira \\
\hline Menta spp. & Hortelã \\
\hline Kalanchoe brasiliensis Cambess & Malva-santa \\
\hline Plectranthus barbatus Andr. & Boldo \\
\hline Rosmarinus officinalis L. & Alecrim \\
\hline \multicolumn{2}{|l|}{ LAURACEAE } \\
\hline Cinnamomum spp. & Canela \\
\hline \multicolumn{2}{|l|}{ LYTHRACEAE } \\
\hline Punica granatum $\mathrm{L}$. & Romã \\
\hline \multicolumn{2}{|l|}{ MALVACEAE } \\
\hline Gossypium spp. & Algodão \\
\hline \multicolumn{2}{|l|}{ POACEAE } \\
\hline Cymbopogon citratus Stapf. & Capim-santo \\
\hline \multicolumn{2}{|l|}{ PHYLLANTHACEAE } \\
\hline Phyllanthus niruri L. & Quebra-pedra \\
\hline \multicolumn{2}{|l|}{ RUTACEAE } \\
\hline Citrus sinensis (L.) Osbeck. & Laranjeira \\
\hline Ruta graveolens $\mathrm{L}$. & Arruda \\
\hline
\end{tabular}




\begin{tabular}{|l|c|}
\hline XANTHORRHOEACEAE & Babosa \\
\hline Aloe vera $($ L.) Burm. F. & \\
\hline
\end{tabular}

Fonte: Própria (2019).

Os entrevistados foram questionados sobre quais das plantas medicinais anteriormente citadas por eles são endêmicas da Caatinga, e Capim-santo (8 citações), Erva doce (6), Alecrim (6), Boldo (6), Aroeira (5), Babosa (5) e Hortelã (4), foram as mais frequentes. No entanto, foi possível verificar que dentre essas plantas apenas a aroeira (Myracrodruon urundeuva (Alemão) Engl.) é endêmica da Caatinga, o que demonstrou um número relativamente baixo de espécies nativas em relação as demais mencionadas. Com esse resultado percebe-se que há uma carência de conhecimentos sobre as plantas medicinais endêmicas do bioma.

Para a frequência de uso dessas plantas, foi possível observar que os participantes raramente utilizam $(45,45 \%)$, (Gráfico 1). Resultado semelhante a esse foi encontrado no trabalho de Brasileiro et al. (2008), em que boa parte dos entrevistados também afirmaram que raramente $(55,47 \%)$ utilizam as plantas medicinais.

Gráfico 1. Distribuição da frequência de uso das plantas medicinais mencionadas pelos estudantes da Escola EREM Desembargador João Paes, Serrita/PE.

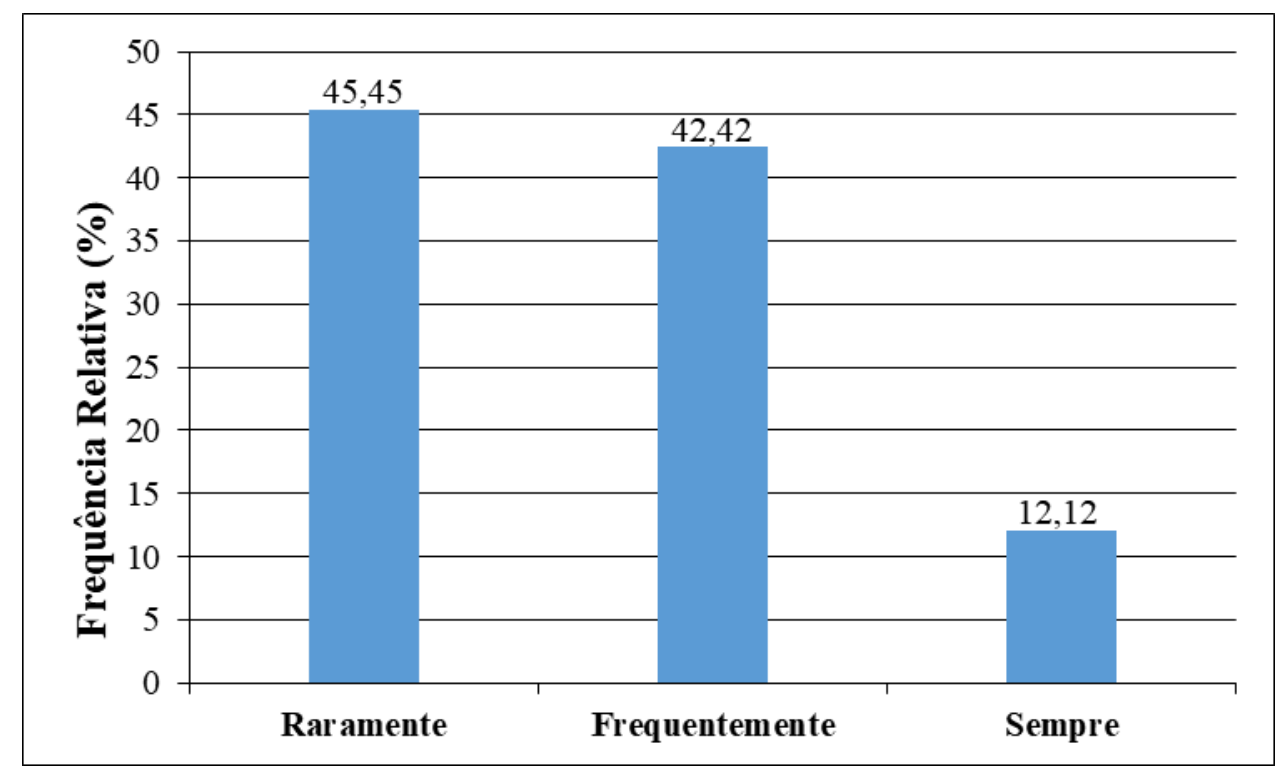

Fonte: Própria (2019).

Ao serem questionados acerca do resultado obtido após o uso das plantas medicinais, todos afirmaram que houve efeito positivo. Observa-se que esse resultado difere do que foi apresentado no estudo de Vieira e Leite (2018), em que dois dos trinta entrevistados disseram que não obtiveram resultados da diminuição dos sintomas da doença.

Para as formas de uso, pode-se constatar que o chá $(71,11 \%)$ seguido de xarope 
$(24,44 \%)$ foram citados com maior frequência, (Gráfico 2). Esses dados também foram encontrados por Mosca e Loiola (2009), em que observou-se a predominância dos chás e xaropes para as formas de preparo das plantas medicinais. As autoras supracitadas pressupõem que esses resultados estão relacionados com a facilidade na preparação dos remédios.

Gráfico 2. Representatividade das formas de uso das espécies botânicas mencionadas pelos estudantes da Escola EREM Desembargador João Paes, Serrita/PE.

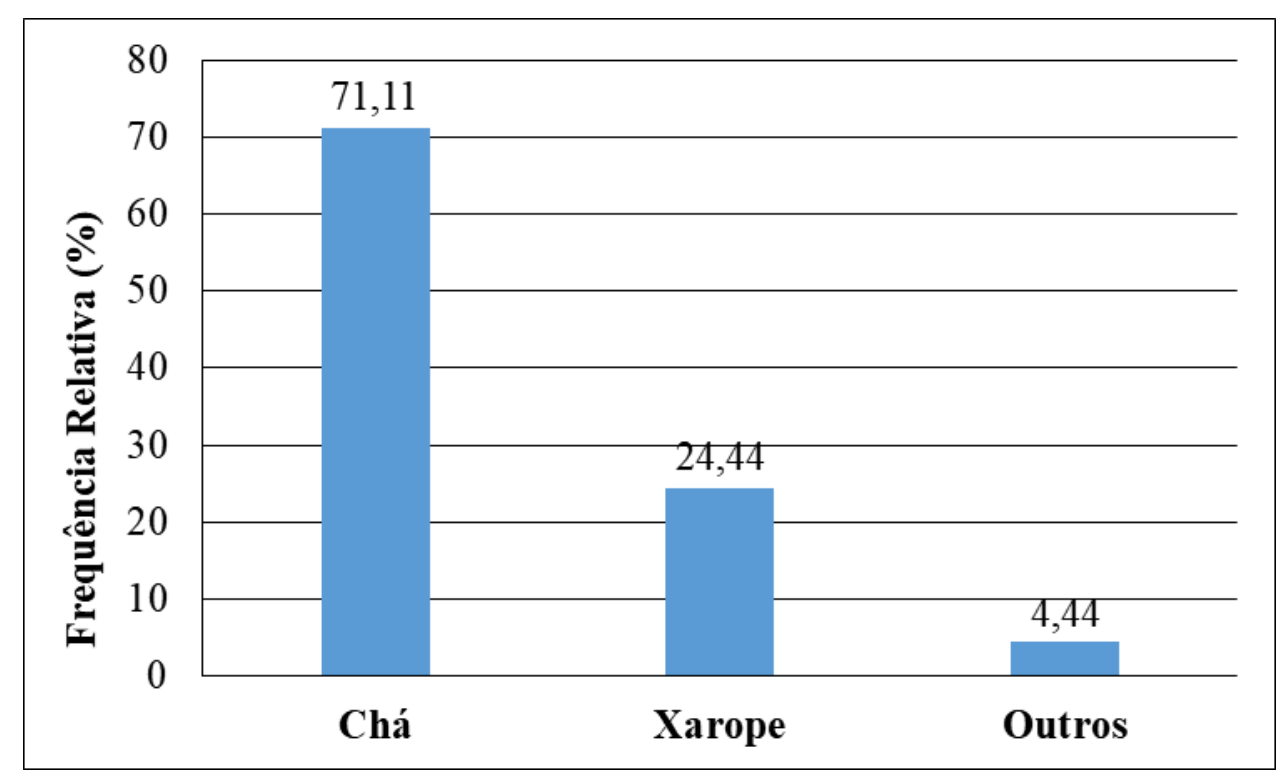

Fonte: Própria (2019).

Quanto a importância das plantas medicinais, todos afirmaram ser para a melhoria da saúde, por serem naturais e de fácil acesso tanto em relação ao local onde são encontradas, quanto na questão financeira, por possuírem um baixo custo e diminuírem os gastos com medicamentos. Além dos benefícios para a saúde, de acordo com Calixto e Ribeiro (2004), as plantas medicinais têm um papel muito importante na questão socioeconômica, tanto para as populações que vivem no meio rural quanto no meio urbano, pois a utilização dessas plantas, na maioria das vezes nativas da sua região ou cultivadas em seu quintal, pode reduzir e/ou eliminar gastos com medicamentos sintéticos.

Segundo Caravaca (2000), o conhecimento sobre as plantas medicinais, tais como a prática de utilização, é uma herança familiar, transmitida de geração a geração. Essa afirmação corroborou com os resultados obtidos, onde os entrevistados afirmaram que as informações acerca das plantas medicinais ocorrem principalmente no meio familiar, sendo transmitidas de geração a geração. 
Com relação à abordagem da aula expositiva e dialogada, foi possível perceber uma significativa participação e interesse dos estudantes durante toda a intervenção, onde os mesmos realizaram com frequência questionamentos e ressaltaram um pouco dos seus conhecimentos acerca da temática. Percebeu-se também que os estudantes demonstraram ainda mais empolgação durante o contato com algumas amostras de plantas medicinais da Caatinga, o que ocasionou a promoção de debates e proporcionou assim interação entre eles, ou seja, a troca de idéias. Desta forma, notou-se uma relevante construção de saberes no que tange as plantas medicinais no geral e principalmente as nativas do bioma Caatinga.

No pós-teste, os entrevistados mencionaram 25 espécies botânicas como as mais utilizadas com fim medicinal, distribuídas em 14 famílias as quais destacaram-se Fabaceae e Lamiacea, (Tabela 2). Dentre essas plantas, a Aroeira (Myracrodruon urundeuva (Alemão) Engl.) (27 citações), o Jucá (Libidibia ferrea (Mart. ex Tul.) L.P. Queiroz var. ferrea) (19), a Babosa (Aloe vera (L.) Burm. F.) (13), o Boldo (Plectranthus barbatus Andr.)(11), o Capimsanto (Cymbopogon citratus Stapf.) (11) e a Ameixa (Ximenia americana L.) (10), tiveram um maior número de citações. Essas espécies foram observadas na lista de principais plantas utilizadas como medicinas em um estudo realizado Souza e Silva (2015). Percebe-se que as famílias que apresentaram maior número de espécies também se destacaram no trabalho de Rodrigo e Andrade (2014).

Tabela 2. Espécies botânicas mencionadas como medicinais pelos estudantes do ensino médio da Escola EREM Desembargador João Paes, Serrita/PE.

\begin{tabular}{|l|c|}
\hline \multicolumn{1}{|c|}{ Família/ Espécie } & Nome Vernáculo \\
\hline AMARANTHACEAE & Mentruz \\
\hline Chenopodium ambosioides & Aroeira \\
\hline ANACARDIACEAE & Coentro \\
\hline Myracrodruon urundeuva (Alemão) Engl. & Erva-doce \\
\hline APIACEAE & Marcela \\
\hline Coriandrum sativum L. & Camomila \\
\hline Pimpinella anisum L. & \\
\hline ASTERACEAE & Imburana-de-cambão \\
\hline Achyrocline satureioides (Lam) DC & \\
\hline Matricaria recutita L. & \\
\hline BURSERACEA & \\
\hline Commiphora leptophloeos (Mart.) J. B. gillett & \\
\hline
\end{tabular}




\begin{tabular}{|c|c|}
\hline \multicolumn{2}{|l|}{ CACTACEAE } \\
\hline Cereus jamacuru DC. & Mandacaru \\
\hline \multicolumn{2}{|l|}{ CANNABACEAE } \\
\hline Cannabis sativa $\mathrm{L}$. & Maconha \\
\hline \multicolumn{2}{|l|}{ FABACEAE } \\
\hline $\begin{array}{l}\text { Anadenanthera colubrina (Vell.) Brenan var. cebil } \\
\text { (Griseb.) }\end{array}$ & Angico \\
\hline Poincianella bracteosa (Tul.) L.P. Queiroz & Catingueira \\
\hline $\begin{array}{l}\text { Libidibia ferrea (Mart. ex Tul.) L.P. Queiroz var. } \\
\text { férrea }\end{array}$ & Jucá \\
\hline Piptadenia stipulacea (Benth.) Burke & Jurema-branca \\
\hline Mimosa tenuiflora (Willd.) Poir. & Jurema-preta \\
\hline Prosopis juliflora (Sw.) DC. & Algaroba \\
\hline \multicolumn{2}{|l|}{ LAMIACEAE } \\
\hline Melissa officinalis L. & Erva-cidreira \\
\hline Menta spp. & Hortelã \\
\hline Kalanchoe brasiliensis Cambess & Malva-santa \\
\hline Plectranthus barbatus Andr. & Boldo \\
\hline Rosmarinus officinalis L. & Alecrim \\
\hline \multicolumn{2}{|l|}{ LYTHRACEAE } \\
\hline Punica granatum $\mathrm{L}$. & Romã \\
\hline \multicolumn{2}{|l|}{ OLACACEAE } \\
\hline Ximenia americana $\mathrm{L}$. & Ameixa \\
\hline \multicolumn{2}{|l|}{ POACEAE } \\
\hline Cymbopogon citratus Stapf. & Capim-santo \\
\hline \multicolumn{2}{|l|}{ RUTACEAE } \\
\hline Citrus sinensis (L.) Osbeck. & Laranjeira \\
\hline \multicolumn{2}{|l|}{ XANTHORRHOEACEAE } \\
\hline Aloe vera $(\mathrm{L}$.$) Burm. F.$ & Babosa \\
\hline
\end{tabular}

Fonte: Própria (2019).

Quando questionados sobre quais das plantas medicinais anteriormente citadas por eles são endêmicas da Caatinga, foram mencionadas a Aroeira (27 citações), o Jucá (17), a Catingueira (9) e a Ameixa (8), com maior frequência. Foi possível observar que as espécies medicinais mais citadas são nativas da Caatinga, o que demonstrou um número relativamente alto quando comparado ao obtido no pré-teste. Com isso, percebe-se que houve um aprendizado significativo em relação as plantas medicinais endêmicas do bioma. Observa-se 
que em um trabalho realizado por Silva e Freire (2010), a maioria das espécies citadas como tendo propriedades medicinais são nativas da Caatinga.

Quanto a relação que se pode estabelecer entre as plantas medicinais e a comunidade, os entrevistados designaram uma ligação de uso e compartilhamento de conhecimentos sobre essas plantas, por serem parte integrante e cultural da comunidade. Esse resultado corrobora com a colocação de Maciel et al. (2002), quando afirmam que o conhecimento acerca das plantas medicinais representa muitas vezes o único meio terapêutico de muitas comunidades e grupos étnicos.

Segundo Freitas e Costa (2011), ao buscar compreender o conhecimento que as pessoas mais velhas detêm, pretende-se refletir sobre a educação bem como as contribuições concebidas pelas as mesmas. Essa afirmação corroborou com os resultados obtidos, onde os entrevistados disseram ser importante a busca de informações a partir dos conhecimentos mais velhos, pois os mesmos dispõem de muitas experiências e saberes que contribuem para o aprendizado de modo geral.

No que se refere a como essas informações podem ajudar no processo de aprendizagem sobre as plantas medicinais, os estudantes afirmaram que são importantes para a obtenção de conhecimentos acerca do uso correto dessas plantas, tais como o preparo, a quantidade e a dosagem. Para Santos e Rosito (2012), a informação e conscientização são essenciais para que o uso das plantas medicinais seja proveitoso, visto que entender as práticas de utilização dessas plantas são fundamentais para obter-se resultados satisfatórios.

\section{Conclusões}

Conclui-se que os estudos etnobotânicos podem fornecer informações valiosas para o reconhecimento e conservação da cultura das plantas medicinais do ecossistema envolvido, uma vez que os estudantes apresentaram um conhecimento razoável sobre essas espécies. Entretanto, ao valer-se desse tema de maneira expositiva e dialogada, foi possível observar uma sensível incorporação desses conhecimentos.

\section{Referências}

BAPTISTA, G. C. S. A Contribuição da etnobiologia para o ensino e a aprendizagem de Ciências: estudo de caso em uma escola pública do Estado da Bahia. Dissertação de mestrado apresentada ao Programa de Pós-graduação em Ensino, Filosofia e História das Ciências, Salvador, Universidade Federal da Bahia - Universidade Estadual de Feira de Santana. 
Salvador-Bahia, p. 2-36, 2007.

BRANCALIONE. L. Educação Ambiental: Refletindo sobre aspectos históricos, legais e sua importância no contexto social. Revista de Educação do IDEAU, v. 11, n. 23, p. 12, 2016.

BRASILEIRO, B. G.; PIZZIOLO, V. R.; MATOS, D. S.; GERMANO, A. M.; JAMAL, C. M. Plantas medicinais utilizadas pela população atendida no "Programa de Saúde da Família", Governador Valadares, MG, Brasil. Revista Brasileira de Ciências farmacêuticas, v. 44, n. 4, p. 629-636, 2008.

CARAVACA, H. Plantas que curam. Editora Virtual Books Online, 2000.

CALIXTO, J. S.; RIBEIRO, E. M. O cerrado como fonte de plantas medicinais para o uso dos moradores de comunidades tradicionais do alto Jequitinhonha, MG. In: Encontro nacional de pós-graduação em ambiente e sociedade, Indaiatuba-MG. Anais, v. 2, 2004.

CNPI - CENTRO NORDESTINO DE INFORMAÇÕES SOBRE PLANTAS. Recife/ PE. Disponível em: http://www.cnip.org.br/bdpn/bd.php?bd=cnip7. Acesso em: 08 set. 2019.

FRANCO, F.; FERREIRA, A. P. N. L.; FERREIRA, M. L. Etnobotânica: aspectos históricos e aplicativos desta ciência. Caderno de Cultura e Ciência, v.10, n.2, p. 17-23, 2011.

FREITAS, S. A.; COSTA, M. J. A identidade social do idoso: memória e cultura popular. Revista Conexão UEPG, v. 7, n. 2, p. 202-211, 2011.

GUARIM-NETO, G. O saber tradicional pantaneiro: as plantas medicinais e a Educação Ambiental. Revista Eletrônica do Mestrado em Educação Ambiental. Rio Grande, v. 17, p. 71-89, 2006.

GUARIM-NETO, G.; SANTANA, S. R.; SILVA, J. B. V. Notas etnobotânicas de espécies de Sapindaceae jussieu. Acta Botânica Brasílica, Feira de Santana, v. 14, n. 3, p. 327-334, 2000.

IBGE. IBGEcidade.ibge.gv.br. Atualizado em 2019. Disponível em: https://cidades.ibge.gov.br/. Acesso em: 29 abril, 2019.

KOVALSKI, M. L.; OBARA, A. T. O estudo da etnobotânica das plantas medicinais na escola. Ciências \& educação (Bauru), v. 19, n. 4, p. 911-927, 2013.

NASCIMENTO, A. P. B. Sobrepeso e obesidade: dieta, nicho alimentar e adaptabilidade em populações humanas rural e urbana de Piracicaba, SP. Tese de Doutorado - Escola Superior de Agricultor Luiz de Queiroz, Piracicaba, p. 96, 2008.

MACIEL, M. A. M.; PINTO, C. A.; VEIGA, J. V. F. Plantas medicinais: a necessidade de estudos multidisciplinares. Química Nova. Rio de Janeiro, v. 25, n. 3, p. 429-438, 2002.

MARTINS, A. G.; ROSÁRIO, D. L.; BARROS, M. N.; JARDIM, M. A. G. Levantamento etnobotânico de plantas medicinais, alimentares e tóxicas da Ilha do Cambu, Município de Belém, Estado do Pará. Revista Brasileira de Farmacologia, v. 86, n.1, p. 21-30, 2005. 
MARTINS, R. C. Plantas medicinais da Caatinga: uso e conhecimento popular em área urbana do Município de Juazeiro-Ba. Monografia (Especialização em educação contextualizada para convivência com o semiárido Brasileiro) - Universidade Estadual da Bahia - UEBA. Juazeiro-Bahia, p. 14-61, 2012.

MEDEIROS, E. T. O.; CRISÓSTIMO, A. L. A importância da aprendizagem das plantas medicinais do ensino da botânica. OS DESAFIOS DA ESCOLA PÚBLICA PARANAENSE NA PERSPECTIVA DO PROFESSOR PDE. Cadernos PDE. Paraná, v.1, 2013.

MERA, J. C. E.; ROSAS, L. V.; LIMA, R. A.; PANTOJA, T. M. A. Conhecimento, percepção e ensino sobre plantas medicinais em duas escolas públicas do Município de Benjamin Constant-AM. Experiência em Ensino de Ciências. V. 13, n. 2, p. 62-79, 2018.

MOSCA, V. P.; LOIOLA, M. I. B. Uso popular de plantas medicinais no Rio Grande do Norte, nordeste Brasil. Revista Caatinga, Mossoró, v. 44, n. 4, p. 225-234, 2009.

OLIVEIRA, F. C.; ALBUQUERQUE, U. P.; FONSECA-KRUEL, V. S.; HANAZAKI, N. Avanços nas pesquisas etnobotânicas do Brasil. Acta Botânica Brasílica, v. 23, n. 2, p. 590$605,2009$.

PAIVA, D. C. C. Atividade anti-inflamatória e antinociceptiva do extrato hidroalcoólico da entrecasca de Pseudobombax marginatum (St. Hill) Rob. Proveniente da Caatinga potiguar. Dissertação (Mestrado em Ciências Naturais). Universidade do Estado do Rio Grande do Norte. Programa de Pós-graduação em CiênciasNaturais. Mossoró-RN, p. 16-65, 2013.

PEREIRA, D.D. Plantas em Prosa e Poesia do Semiárido. Campina Grande: EDUFCG, 2005. 219p.

PILlA, M. A. C.; AMOROZO, M. C. M.; FURLAN, A. Obtenção e uso das plantas medicinais no distrito de Martim Francisco, Município de Mogi-Mirim, SP, Brasil. Acta Botânica Brasílica, v, 20, n. 4, p.789-802, 2006.

PRADO, D. E. As Caatingas da América do Sul. In: LEAL, R. I.; TABARELLI, M.; SILVA, J. M. C. Ecologia e conservação da Caatinga. Recife: Ed. Universitária da UFPE, 2003. $823 p$.

QUEIROZ, M. A. Recursos genéticos vegetais da Caatinga para o desenvolvimento do semiárido brasileiro. Revista Brasileira de Geografia Física, v. 4, n. 6, p. 1135-1150, 2011.

REZENDE, H. A.; COCCO, M. I. M. A utilização de fitoterapia no cotidiano de uma população rural. Revista da Escola de Enfermagem da USP, v. 36, n. 3, p. 28-288, 2002.

RIBEIRO, D. A.; MACÊDO, D. G.; OLIVEIRA, L. G. S.; SARAIVA, M. E.; OLIVEIRA, S. F.; SOUZA, M. M. A.; MENEZES, I. R. A. Potencial terapêutico e uso de plantas medicinais em uma área da Caatinga no estado do Ceará, nordeste do Brasil. Revista Brasileira de Plantas Medicinais, v. 16, n. 4, p. 912-930, 2014. 
RODRIGUES, A. P.; ANDRADE, L. H. C. Levantamento etnobotânico das plantas medicinais utilizadas pela comunidade de Inhamã, Pernambuco, Nordeste do Brasil. Revista Brasileira de Plantas Medicinais, Botucatu, v. 16, n. 3, p. 721-730, 2014.

RODRIGUES, J. S. C. Estudo etnobotânico das plantas aromáticas e medicinais. In: Figueiredo AC, JG Barroso, LG Pedro (Eds), Potencialidades e Aplicações das Plantas Aromáticas e Medicinais. Curso Teórico-Prático, pp. 168-174, $3^{\mathrm{a}}$ Ed., Edição da Faculdade de Ciências da Universidade de Lisboa - Centro de Biotecnologia Vegetal, Lisboa, Portugal, 2007.

SANTOS, B. M. M.; ROSITO, J. M. Uso de plantas medicinais como instrumentos de conscientização: responsabilidade social e ambiental. Monografias Ambientais, v. 7, n. 7, p. 1478-1491, 2012.

SILVA, M. R. A utilização do conhecimento de plantas medicinais como ferramenta para estimular a preservação ambiental. Monografias Ambientais, v. 6, n. 6, p. 1354-1380, 2012.

SILVA, T. S.; FREIRE, E. M. X. Abordagem etnobotânica sobre plantas medicinais citadas por populações do entorno de uma unidade de conservação da caatinga do Rio Grande do Note, Brasil. Revista Brasileira de Plantas Medicinais, Botucatu, v. 12, n. 4, p. 427-435, 2010 .

SOUZA, V. C.; LORENZI, H. Botânica Sistemática: guia ilustrado para identificação das famílias Fanerógamas nativas e exóticas no Brasil, baseado em APG II. Nova Odessa, SP: Instituto Plantarum, 2008.

SOUZA, M. V. F.; SILVA, J. M. A. Consumo de plantas medicinais por mulheres idosas no município de Quixeré-CE. Revista Baiana de Saúde Pública, v. 39; n. 3, p. 552-569, 2015.

VEIGA, J. V. F.; PINTO, A. C. Plantas medicinais: cura segura?. Química Nova, v. 28, n. 3, p. 519-528, 2005.

VIEIRA, V. D.; LEITE, L. M. S. O uso do conhecimento popular das plantas medicinais utilizadas pela comunidade no Nordeste. Temas em Saúde, p. 876-890, 2018.

\section{Apêndices}

Apêndice A - Entrevista semiestruturada apresentada como pré-teste aos discentes do $2^{\circ}$ Ano A do Ensino Médio.

\begin{tabular}{|c|c|}
\hline 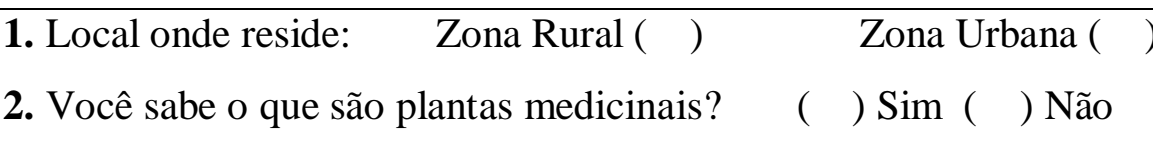 & \\
\hline 3. Você e/ou algum familiar faz (em) uso das plantas medicinais?( & ) $\operatorname{Sim}(\quad)$ \\
\hline
\end{tabular}


5. Quais as plantas mais utilizadas com fim medicinal?

6. Das plantas citadas na questão anterior, quais são nativas (endêmicas) da Caatinga?

7. Com que frequência são utilizadas?

( ) Nunca ( ) Raramente ( ) Frequentemente ( ) Sempre

8. O uso teve efeito positivo? ( ) Sim ( ) Não

9. Quais as formas de uso?
( ) Chá
( ) Pomada
( ) Xarope
( ) Outros:

10. Qual a importância das plantas medicinais?

11. Como as informações sobre essas plantas medicinais são transmitidas?

Fonte: Própria (2019). 
Apêndice B - Entrevista semiestruturada apresentada como pós-teste aos discentes do $2^{\circ}$ Ano A do Ensino Médio.

1. Para você, quais as plantas mais utilizadas com fim medicinal?

2. Das plantas citadas na questão anterior, quais são nativas (endêmicas) da Caatinga?

3. Que relações podemos estabelecer entre as plantas medicinais e a sua comunidade?

4. Para você, qual a importância da busca de informações a partir dos conhecimentos dos mais velhos?

5. Como essas informações podem ajudar no processo de aprendizagem sobre as plantas medicinais?

Fonte: Própria (2019). 